\title{
Modern Technology for Acupuncture Research: A Short Review from the Medical University of Graz
}

\author{
Lu Wang, Gerhard Litscher* \\ Research Unit of Biomedical Engineering in Anesthesia and Intensive Care Medicine and TCM \\ Research Center Graz, Medical University of Graz, Graz, Austria \\ Email: \{lu.wang, gerhard.litscher\}@medunigraz.at \\ Received December 4, 2010; revised December 8, 2010; accepted December 10, 2010
}

\begin{abstract}
This short review article gives a brief description of the TCM Research Center Graz, especially the part of high-tech acupuncture research. A new technology for non-invasive acupuncture stimulation using violet laser $(405 \mathrm{~nm}, 110 \mathrm{~mW}, 500 \mu \mathrm{m})$ is described. With this kind of laser it is possible to induce a so-called Deqi sensation which is very important in Chinese acupuncture. The second part of the review paper describes the new teleacupuncture which has been performed for the first time between the China Academy of Chinese Medical Sciences and the TCM Research Center Graz at the Medical University of Graz.
\end{abstract}

Keywords: Violet Laser Acupuncture, Teleacupuncture, Sino-Austrian Research, Biomedical Engineering

\section{Introduction-TCM Research Center Graz}

Many people take the different applications of the laser in medicine for granted. Although the invention of the laser is celebrating its 50th anniversary in 2010 [1], there are still some areas in traditional medicine that require intensive translational laser research. One of these areas is innovative laser acupuncture [2].

From acupuncture to the many hundreds of different medical herbs-traditional Chinese medicine (TCM) is booming. And it is effective: TCM has been practiced with great success for more than 4,000 years, and the Western demand for something to complement classical Western medicine has been increasing for years. Graz plays a central role in the study of TCM in Europe. In March 2007 the "Research Center for Traditional Chinese Medicine" was established, and subsequently a global high-tech acupuncture network developed (see Figure 1), which integrates the various national and international activities in research and teaching. KarlFranzens-University Graz and the Medical University of Graz are the two Austrian universities in the forefront.

The Research Unit of Biomedical Engineering in Anesthesia and Intensive Care Medicine at the Medical University of Graz (http://litscher.info) has been dealing with high-tech acupuncture research for over 13 years. In this case, evidence-based, scientific work is the foundation. Consequently, all research is carried out on the ba- sis of scientific methods (comp. Figure 2) [3-12]. Special focus is placed on basic research and those aspects of TCM, which until now got paid only little attention. Beside needle acupuncture, both laser- and electroacupuncture play a very important role. In addition, first transcontinental teleacupuncture measurements including laser acupuncture are realized, which means the patient is in China and the high-tech analysis is conducted in Graz via internet connections.

The importance of TCM has grown in recent years. About seventy percent of the Austrians want classical Western medicine to be supplemented by complementary medical services. Similar data exist in Switzerland and Germany. The acceptance of TCM is particularly high, with an approval rate of eighty percent. However, not

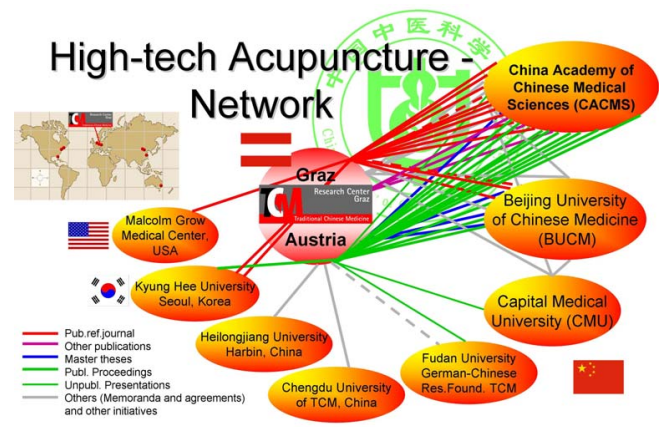

Figure 1. High-tech acupuncture network of the TCM Research Center Graz. 


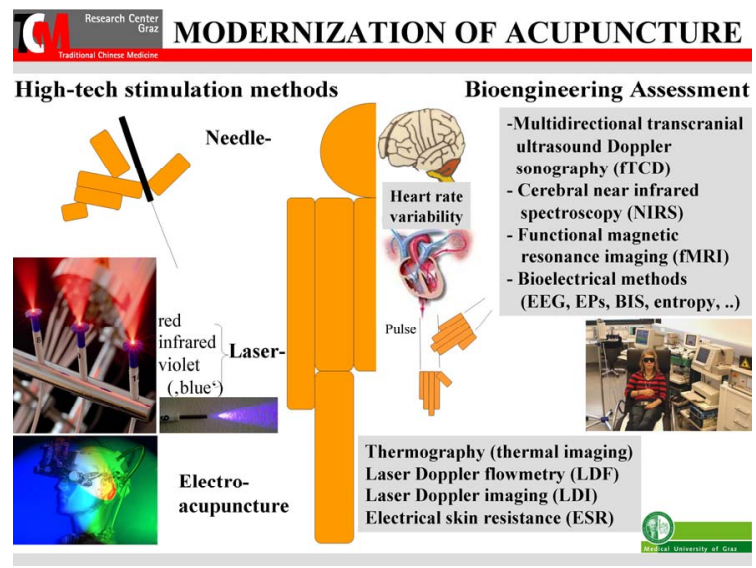

Figure 2. Modernization of acupuncture (modified from [13]).

only the population, even the doctors show a growing interest. For Graz as a location of research, the establishment of the competence Center will have long-term positive impacts: already existing cooperations with other institutions that conduct research in the field of TCM, will be amplified, and the know-how transfer between Austria and China will also be intensified.

\section{Fascination "Violet Laser Acupuncture"}

Only recently the first scientific results concerning a new method of acupuncture, the 'violet laser needle acupuncture', were presented. It is possible to achieve significant effects such as increases in blood flow in small vessels with the violet wavelength of $405 \mathrm{~nm}$ (Figure 3) and a specially focused beam at the acupuncture point, despite low penetration depth.

Within a cooperative research project between Austria and China, first scientific results on this new violet laser acupuncture method were published [13,14]. In addition, the new violet laser can trigger a Deqi feeling, usually typical for needle acupuncture, which often manifests itself as a mild electric tingling.

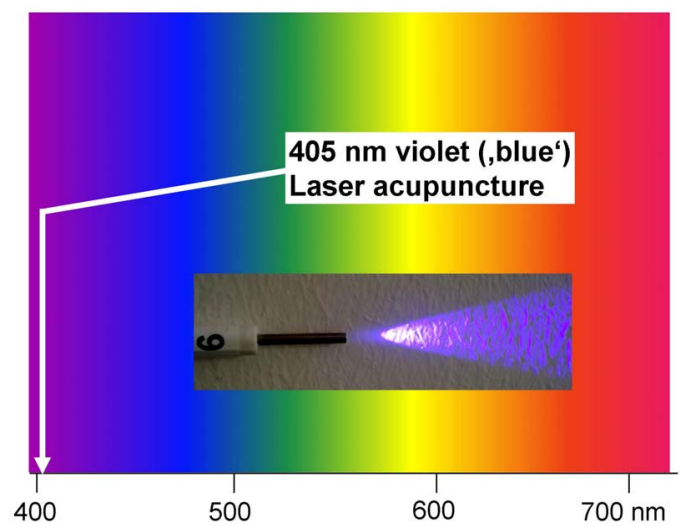

Figure 3. Violet laser acupuncture needle (modified from [2]).
For Chinese people, this Deqi feeling is an important prerequisite for a successful acupuncture treatment. While using a red or infrared laser that kind of stimulus is not immediately felt, this is different using the violet laser. Thirteen adult Chinese volunteers reported an immediately felt stimulus, similar to a Deqi feeling evoked by a metal needle. "The effect is comparatively not as strong as an ant bite, but longer-lasting," said a Chinese female volunteer. If the acupuncture point Neiguan was stimulated, there was a significant decrease in heart rate associated with a pleasant, stress-reducing experience. This particular point, which is also referred to as "Pericard 6" and is located near the wrist crease, is said to achieve a circulatory-regulating effect according to TCM. These effects did not occur in a control study in which the violet laser was turned off. Further investigations, concerning the question whether this effect is also present in Europeans in the same amount, are in progress. In the future the procedure might be used in patients with high blood pressure or generally for age-related diseases of the cardiovascular system as an additive gentle, regulatory method. Before that, however, basic research studies have to be carried out.

\section{Teleacupuncture-Patient in China, Analysis in Austria}

The transcontinental teleacupuncture is even more spectacular. The first studies have been carried out successfully: 24-hour recordings of the electrical activity of the heart were registered in patients in China with a system partially developed in Graz, and immediately after the acupuncture treatment the data were sent from a bedside computer in Beijing over a distance of $7650 \mathrm{~km}$ via Internet for computer analysis at the Medical University of Graz. The acupuncturists in China were informed of the results immediately. The autonomic nervous system plays a central role in the current investigations. Computer-based heart rate and heart rate variability measurements are important variables.

Already the medical scholar Wang Shu-Ho carried out observations in China ( 220 BC.) that are directly related to heart rate variability (HRV) and the autonomic nervous system. He noted: "If the heartbeat becomes as regular as the beating of the woodpecker or the dripping of rain on the roof the patient will be dead within four days." The 'health indicator'-variable heart rate-is therefore already very well known, but found its way into 'modern' science only in the last third of the last century, moving into many areas of medicine.

By definition, HRV represents percentage changes of consecutive ventricular complexes (RR intervals) in the electrocardiogram (ECG), and is modulated by the blood-pressure control system, influences from the hy- 


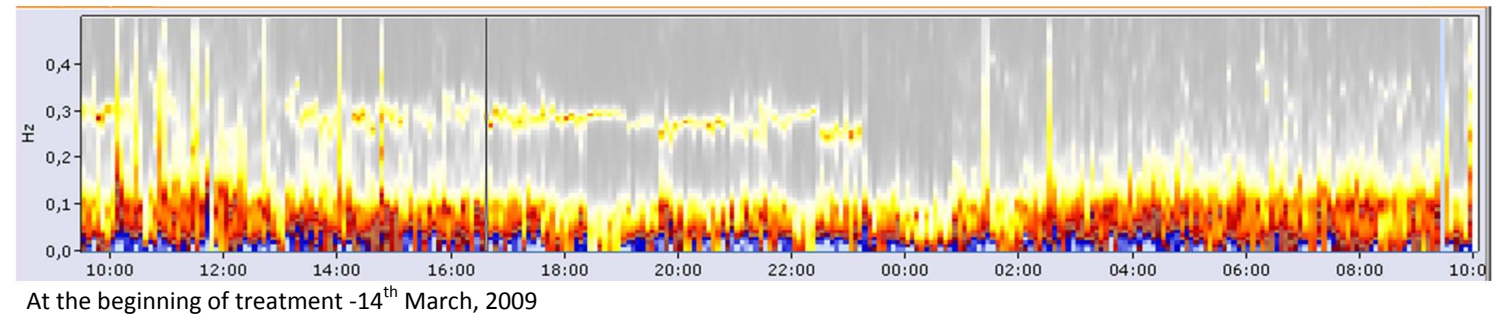

A

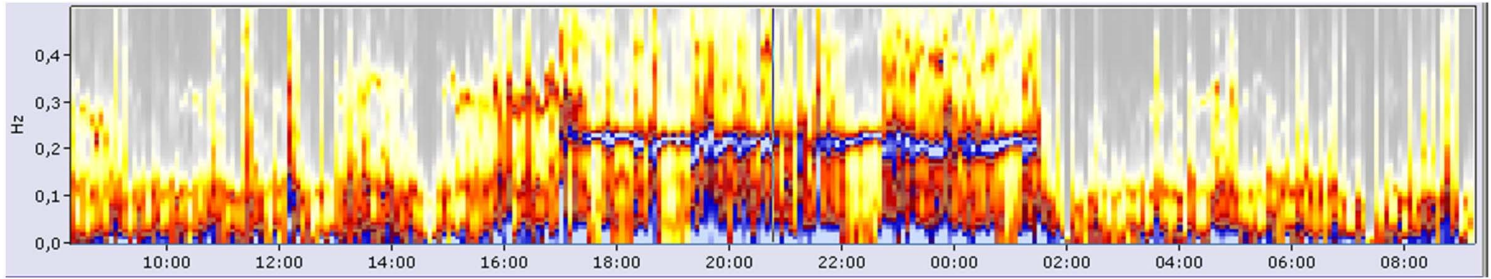

After four acupuncture sessions- $30^{\text {th }}$ March, 2009

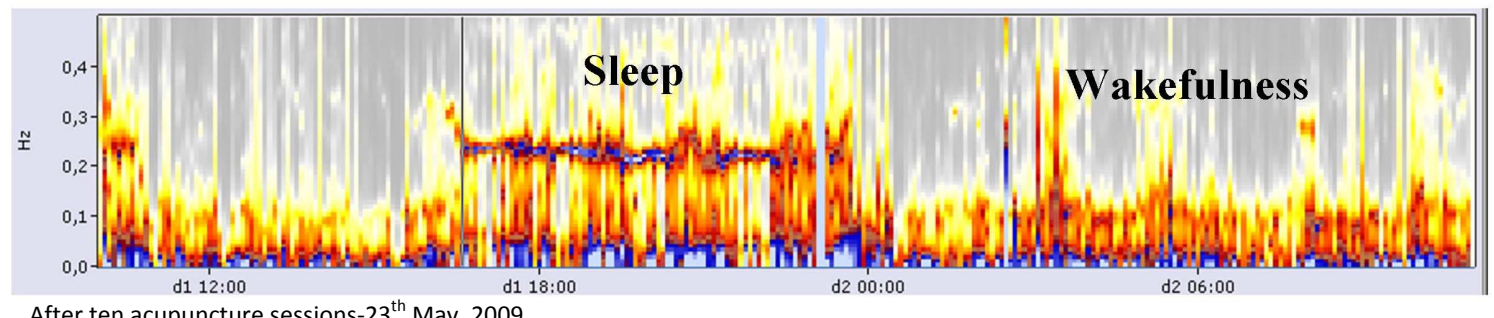

Figure 4. First teleacupuncture measurements between Europe (Graz) and Asia (Beijing). The graphs show sympathetic and vagal activity, and this can be used to indicate the patient's health and quality of sleep (modified from [15,16]).

pothalamus and especially by the vagal part of centers in the lower brain stem.

Figure 4 shows the improvement in health (sleepwake cycle) of a 31-year-old female patient from Beijing over the course of more than two months. At the beginning of treatment (A) the sleep-wake cycle is not clearly marked. After four acupuncture treatments using needle and laser needle acupuncture (B), there is a clearly marked sinus arrhythmia (middle) in the sleep state ( 0.2 to $0.3 \mathrm{~Hz}$ frequency). After ten acupuncture treatments (C) this standard pattern has become stable. For the first time, the therapeutic effect of acupuncture can be visualized not only subjectively, as described by the patient, but with computer-based objective data $[15,16]$.

All further developments of teleacupuncture clearly show that by cutting out lines of communication, omission of redundant research studies as well as simplification of the diagnostic and therapeutic process ultimately not only are costs saved but also time is gained.

The joint research on laser acupuncture between Europe and China shows one thing clearly: Bridging the gap between Eastern and Western medicine has become reality thanks to modern technology. Now we need to make the resulting opportunities and outcomes available to all parties.

\section{Acknowledgements}

The authors thank Ms. Ingrid Gaischek, MSc (Research Unit of Biomedical Engineering in Anesthesia and Intensive Care Medicine and TCM Research Center Graz, Medical University of Graz), Ms. Xie Zheng, MD, (Beijing University of Chinese Medicine and Medical University of Graz) and Prof. Zhang Weibo, $\mathrm{PhD}$, and Ms. Tao Huang, MD PhD (both from the China Academy of Chinese Medical Sciences).

The investigations were supported by the Austrian Federal Ministries of Science and Research and of Health and the Eurasia Pacific Uninet (project 'Bioengineering and clinical assessment of high-tech acupuncture -a Sino-Austrian research pilot study'), are also part of the project 13463 of the Jubiläumsfonds of the Oesterreichische Nationalbank (OeNB) and are supported by the Department of Science of the City of Graz. The measurements were performed within the areas 'Sustainable Health Research' and 'Neuroscience' at the Medical University Graz. 
This paper was presented in part at the Laser Helsinki Congress 2010 (Aug 21 ${ }^{\text {st }}, 2010$ ) by the corresponding author.

\section{References}

[1] N. Anscombe and O. Graydon, “50 $0^{\text {th }}$ Anniversary,” Nature Photonics, Vol. 4, 2010, 279.

[2] G. Litscher, T. Huang, L. Wang and W. B. Zhang, "Violet Laser Acupuncture - Part 1: Effects on Brain Circulation," Journal of Acupuncture Meridian Studies, Vol. 3, No. 4, 2010, pp. 255-259.

[3] G. Litscher, "Bioengineering Assessment of Acupuncture, Part 1. Thermography," Critical Reviews in Biomedical Engineering, Vol. 34, No. 1, 2006, pp. 1-22.

[4] G. Litscher, "Bioengineering Assessment of Acupuncture, Part 2: Monitoring of Microcirculation," Crit Rev Biomed Eng, Vol. 34, No. 4, 2006, pp. 273-294.

[5] G. Litscher, "Bioengineering Assessment of Acupuncture, Part 3: Ultrasound," Critical Reviews in Biomedical Engineering, Vol.34, No.4, 2006, pp. 295-326.

[6] G. Litscher, "Bioengineering Assessment of Acupuncture, part 4: Functional Magnetic Resonance Imaging," Critical Reviews in Biomedical Engineering, Vol. 34, No. 4, 2006, pp. 327-345.

[7] G. Litscher, "Bioengineering Assessment of Acupuncture, Part 5: Cerebral Near Infrared Spectroscopy,” Critical Reviews in Biomedical Engineering, Vol.34, No.6, 2006, pp. 439-457.

[8] G. Litscher, "Bioengineering Assessment of Acupuncture, Part 6: Monitoring-Neurophysiology," Critical Reviews in Biomedical Engineering, Vol.35, No.1, 2007, pp. 1-38.
[9] G. Litscher, "Bioengineering Assessment of Acupuncture, Part 7: Heart Rate Variability," Critical Reviews in Biomedical Engineering, Vol.35, No.3, 2007, pp. 183-195.

[10] G. Litscher, "Bioengineering Assessment of Acupuncture, Part 8: Innovative Moxibustion," Critical Reviews in Biomedical Engineering, Vol.38, No.3, 2010, pp. 117-126.

[11] G. Litscher, "Ten Years Evidence-Based High-Tech Acupuncture-A Short Review of Peripherally Measured Effects," Evidence-based Complementary Alternative Medicine, Vol. 6, No. 2, 2009, pp. 153-158.

[12] G. Litscher, “Ten Years Evidence-Based High-Tech Acupuncture-A Short Review of Centrally Measured Effects (part II)," Evidence-based Complementary Alternative Medicine, Vol. 6, No. 3, 2009, pp. 305-314.

[13] G. Litscher, "Modernization of Traditional Acupuncture using Multimodal Computer-Based High-Tech MethodsRecent Results of Blue Laser and Teleacupuncture from the Medical University of Graz," Journal of Acupuncture Meridian Studies, Vol. 2, No. 3, 2009, pp. 202-209.

[14] G. Litscher, L. Wang, Z. Xie and I. Gaischek, "Blauer Laser in der Akupunktur-ein Erster Review Report," Schweiz Z Ganzheitsmed, Vol. 22, 2010, pp. 167-170.

[15] G. Litscher, "Transkontinentale Akupunkturforschung mittels Teleakupunktur,” Promed komplementär, Vol. 16, No. 3, 2009, pp. 8-11.

[16] G. Litscher and Asian-Austrian High-tech Acupuncture Research Network, "Transcontinental and Translational High-Tech Acupuncture Research Using ComputerBased Heart Rate and 'Fire of Life' Heart Rate Variability Analysis,” Journal of Acupuncture Meridian Studies, Vol. 3, No. 3, 2010, pp. 156-164. 